\title{
Closed Form Solution of Synchronous Machine Short Circuit Transients
}

\author{
Gibson H.M. Sianipar \\ School of Electrical Engineering and Informatics, \\ Bandung Institute of Technology
}

\begin{abstract}
This paper presents the closed form solution of the synchronous machine transients undergoing short circuit. That analytic formulation has been derived based on linearity and balanced conditions of the fault. Even though restrictive, the proposed method will serve somehow or other as a new resource for EMTP productivity. Indisputably superior, the closed-form formulation has some features inimitable by discretization such as continuity, accuracy and absolute numerical stability. Moreover, it enables us to calculate states at one specific instant independent of previous states or a snapshot, which any discretization methods cannot do.
\end{abstract}

Keywords: synchronous machine; short circuit transients; dq-domain model; state space formulation; closed form solution; superposition.

\section{$1 \quad$ Introduction}

The solution of differential equations of the power system components, such as machines, transformers, reactors and lines, in electromagnetic transient or transient stability programs has been so far based on numerical discretization [1,2-10]. The fact that many power system components have nonlinear, time varying, frequency dependent or distributed parameters seems to be the reason. However, depending on the time scale and thus frequency bandwith involved, the models may be ranging from a highly detailed one that includes frequency dependent and/or time delay parameter as found in traveling wave phenomena in a long transmission line up to a simple power frequency lumped parameter one as found in the study of short circuit transients in machines. This paper focuses on the latter.

The solution approach almost common to nowadays electromagnetic transients program is based on Dommel multi nodal method [2]. In this approach each lumped parameter is discretized individually, using trapezoidal rule [1], to create a linear relation between current and voltage at a given time $t$ plus a known constant representing historical current/voltage at the time $\mathrm{t}-\Delta \mathrm{t}$. The set of all components current/voltage linear relations are assembled, according to Kirchoff law, in one nodal matrix equation, preferably in conductance form as it offers sparsity that can be benefitted to save space and to speed up calculation.

Received February $10^{\text {th }}, 2010$, Revised March $23^{\text {rd }}, 2010$, Accepted for publication March $30^{\text {th }}, 2010$. 
The resulting system of linear equations is solved for all nodal voltages at time t. That procedure is iterated at every time step up to the end of simulation.

When all components are linear, the resulting nodal conductance matrix needs to be asssembled and decomposed only once at the initial stage. The situation is different for nonlinear component where the coefficient of the linearized current and voltage relation may change from one time step to another. Therefore, in order not to rebuild and refactor the conductance matrix at every time step, each nonlinear component is represented separately as a current source using compensation theory [3].

The most time consuming part in the discretization based method is the obligation to solve the resulting system of linear equations, with a computation time that may grow from at best as $\mathrm{O}(\mathrm{b})$, where $\mathrm{b}$ is the number of network branches, up to the worst as $\mathrm{O}\left(\mathrm{n}^{3}\right)$, where $\mathrm{n}$ is the number of nodes, at each time step. When the time step chosen is too small for better accuracy and stability, the total computation time may be unfeasibly very long. This is the main reason why the discretization based method so far has been unable to solve electromagnetic transients in a power system of real size within reasonable time.

According to Sianipar [11], in power system with linear network and independent sources, an exact solution to short circuit transients can be obtained using superposition theory. It was shown that the procedure to find a partial solution for each source working alone in the network one at a time can be made efficient using a two-port equivalent concept. In it the network is reduced to equivalent impedance as seen by a source working alone, with other sources zeroed, in the faulted network. However, the source model used was too simple that is an EMF behind an impedance so that the result may not be sufficiently accurate to simulate the real phenomenon. In this paper, as a representative, a synchronous machine, which is the most complex among sources, is to be modeled in details describing all stator and rotor windings transients. As an independent and important topic, this paper is dedicated solely to the solution of the short circuit transients in the single machine, while extension to the multi machines case will be reported in a companion paper.

The modeling of a synchronous machine in the electro- magnetic transients program has been developing for quite a long time. Offering simplicity, the dqdomain modeling has been dominating the electromagnetic transients program [1-3,5] or EMTP up to the recent challenges from phase-domain modeling [69]. Even there was a claim of its incorrectness in unbalanced case [6] that had boldly been refuted in $[4,10]$. Creating time varying parameters, phase-domain modeling turns out to be more complicated and omits for good the possibility of analytic solution as presented in this paper. 
The main contribution of this paper is the derivation of the closed form solution, abbreviated to CS, to synchronous machine differential equations. A closed form solution of the short circuit transients in a machine will open the possibility of calculating the machine states at one particular instant or a snapshot. In some applications, like short circuit study for determining circuit breaker capacity or for relays coordination, this specific CS feature is very welcome as it offers fast calculation even system wisely. There is no more need for the time-consuming calculation of the previous consecutive states as in the discretization based method. Even in a continuous simulation the proposed method has a clear advantage in faster calculation speeds and more accurate results. Besides, it resolves definitely the numerical stability problem.

This closed form solution as shown in this paper is derivable as long as we are limiting the problem to linear and balanced conditions. The linearity assumption will give conservative results because nonlinearity such as saturation almost invariably tends to increase damping [5,7]. In some applications like determining circuit-breaker interrupting capacity, slightly conservative result is preferable to faithful but hard-earned one. The inclusion of non linearity and unbalanced conditions are to be left for future.

\section{The Formulation}

\subsection{Synchronous Machine In Dq-Domain Modeling}

By following motor convention and q-axis leading d-axis by $90^{\circ}$, the differential and algebraic equations governing the relationship of the machine terminal voltages, currents and flux linkages in dq-domain model can be written as follows

$$
\begin{aligned}
& p \boldsymbol{\psi}+\boldsymbol{\Omega} \cdot \boldsymbol{\Psi}+\boldsymbol{R} \cdot \boldsymbol{i}=\boldsymbol{V} \\
& \boldsymbol{\psi}=\boldsymbol{L} \cdot \boldsymbol{i}
\end{aligned}
$$

where

$$
\begin{aligned}
& \boldsymbol{\Psi}=\left[\begin{array}{lllllll}
\psi_{d} & \psi_{q} & \psi_{0} & \psi_{f} & \psi_{h} & \psi_{g} & \psi_{k}
\end{array}\right] \\
& \boldsymbol{v}=\left[\begin{array}{lllllll}
v_{d} & v_{q} & v_{0} & v_{f} & v_{h} & v_{g} & v_{k}
\end{array}\right]^{t} \\
& \boldsymbol{i}=\left[\begin{array}{lllllll}
i_{d} & i_{q} & i_{0} & i_{f} & i_{h} & i_{g} & i_{k}
\end{array}\right]^{t}
\end{aligned}
$$

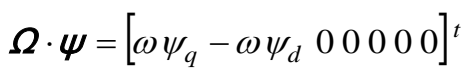


Note : $\Omega$ is a 7 by 7 matrix of zero entries except:

$$
\begin{aligned}
& \Omega_{1,2}=\omega \quad \Omega_{2,1}=-\omega \\
& \mathbf{R}=\operatorname{Diag}\left[\begin{array}{lllllll}
\mathrm{R}_{\mathrm{a}} & \mathrm{R}_{\mathrm{a}} & \mathrm{R}_{\mathrm{a}} & \mathrm{R}_{\mathrm{f}} & \mathrm{R}_{\mathrm{h}} & \mathrm{R}_{\mathrm{g}} & \mathrm{R}_{\mathrm{k}}
\end{array}\right] \\
& L=\left[\begin{array}{ccccccc}
L_{d} & \cdot & \cdot & L_{a f} & L_{a h} & \cdot & \cdot \\
\cdot & L_{q} & \cdot & \cdot & \cdot & L_{a g} & L_{a k} \\
\cdot & \cdot & L_{0} & \cdot & \cdot & \cdot & \cdot \\
L_{a f} & \cdot & \cdot & L_{f} & L_{f h} & \cdot & \cdot \\
L_{a h} & \cdot & \cdot & L_{f h} & L_{h} & \cdot & \cdot \\
\cdot & L_{a g} & \cdot & \cdot & \cdot & L_{g} & L_{g k} \\
\cdot & L_{a k} & \cdot & \cdot & \cdot & L_{g k} & L_{k}
\end{array}\right]
\end{aligned}
$$

Here we don't represent the dynamic of the mechanical part because its time constant is very much larger than the electromagnetic time constants.

With linearity assumption of the flux linkage and current relationship we can rewrite (1) as:

$$
L \cdot p i+(\Omega \cdot L+R) \cdot i=V
$$

where $p$ is a differential operator.

Symbols in bold signify vector or matrix. The following symbols $\psi, v, i$ stand for flux linkage, voltage and current and $R$ and $L$ for resistance and inductance, respectively. Subscripts $\mathrm{f}$ and $\mathrm{h}$ stand for the field and d-axis damper windings while subscripts $\mathrm{g}$ and $\mathrm{h}$ stand for the eddy current and $\mathrm{q}$-axis damper windings respectively.

In this paper, we limit the problem to the balanced fault case. In dq-domain either the resistance or the inductance matrix of the fault is diagonal. Therefore, without losing generality, we can write them as follows

$$
\boldsymbol{R}^{f}=\operatorname{Diag}\left(R_{d}^{f} R_{q}^{f} R_{o}^{f}\right) \text { and } \boldsymbol{L}^{f}=\operatorname{Diag}\left(L_{d}^{f} L_{q}^{f} L_{o}^{f}\right)
$$

Since the d- or q- or o-axis currents in the machine and the fault are respectively the same but of opposite reference directions, then, the relationship between terminal voltages and currents in the fault can be written as

$$
\boldsymbol{V}_{s}=-\boldsymbol{R}^{\boldsymbol{f}} \boldsymbol{i}_{s}^{f}-\boldsymbol{L}^{\boldsymbol{f}} p \boldsymbol{i}_{s}^{f}
$$

where $\boldsymbol{v}_{s}=\left[v_{d} v_{q} v_{o}\right]^{t}$ and $\boldsymbol{i}_{s}=\left[\begin{array}{lll}i_{d} & i_{q} & i_{o}\end{array}\right]^{t}$. 
In the rotor all damper windings are shorted and the field winding is energized by a dc source $E_{f}$, then, the rotor voltage vector can be written as

$$
\boldsymbol{v}_{r}=\left\lfloor\begin{array}{llll}
E_{f} & 0 & 0 & 0
\end{array}\right]
$$

The subscripts $s$ and $r$ in (10) and (11) designate stator and rotor sub-vectors.

\subsection{Composition of Fault and Machine}

In order to be able to solve (9) in a closed form we need to remove all time dependent components from vector $\boldsymbol{v}$. Those components as shown in (10) are present only on stator side. How can that condition be achieved? A simple way out: we need only to assume that the resistances and inductances of the fault as an integral part of the machine. In fact, electrically they are indeed, as they can be taken as part of the stator resistances and stator leakage inductances. By joining the fault resistances and inductances to their respective machine resistances and inductances to create composite resistances and inductances then we can look now the stator terminals as being shorted. Therefore, values of $v_{d}, v_{q}$ and $v_{0}$ of the vector $\boldsymbol{v}$ are zero. In so doing, (9) can now be rewritten as

$$
\boldsymbol{L}^{c o m} \cdot p \boldsymbol{i}+\left(\Omega \cdot \boldsymbol{L}^{\mathrm{com}}+\boldsymbol{R}^{\mathrm{com}}\right) \cdot \boldsymbol{i}=E_{f} \boldsymbol{e}_{f}
$$

where $\boldsymbol{e}_{f}=\left[\begin{array}{llllll}0 & 0 & 0 & 1 & 0 & 0\end{array}\right]^{t}$, and $\boldsymbol{R}^{\text {com }}$ and $\boldsymbol{L}^{\text {com }}$ are made up basically of $\boldsymbol{R}$ and $L$ respectively with the following elements adjusted: $R_{s}^{c o m}=R_{s}+R_{s}^{f}$ and $L_{s}^{c o m}=L_{s}+L_{s}^{f}$ for $s=d, q, 0$.

By rearrangement, (12) can be put into the following standard state space form

$$
p \boldsymbol{i}=\boldsymbol{A} \cdot \boldsymbol{i}+\boldsymbol{u}
$$

where

$$
\boldsymbol{A}=-\left(\boldsymbol{L}^{\mathrm{com}}\right)^{-1}\left(\Omega \cdot \boldsymbol{L}^{\mathrm{com}}+\boldsymbol{R}^{\mathrm{com}}\right)
$$

and

$$
\boldsymbol{u}=\left(\boldsymbol{L}^{\mathrm{com}}\right)^{-1} E_{f} \boldsymbol{e}_{f}
$$

Matrix $\boldsymbol{A}$ being time invariant, so (13) is solvable in a closed form. 


\subsection{Solution in Closed Form}

In [12], Kundur presented a procedure based on closed form solution for calculating zero input, $\boldsymbol{u}=\boldsymbol{O}$, response of (13).

Below, the procedure is extended to a constant non-zero input response.

Diagonalize $\boldsymbol{A}$ using orthogonal transformation

$$
\Lambda=\boldsymbol{V}^{-1} \cdot \boldsymbol{A} \cdot \boldsymbol{V}
$$

where $\Lambda=\operatorname{Diag}\left[\lambda_{1}, \lambda_{2}, \lambda_{3}, \lambda_{4}, \lambda_{5}, \lambda_{6}, \lambda_{7}\right]$ is the diagonal matrix of eigenvalues and $V$ is the matrix of eigenvectors.

Using (16) substitute $\boldsymbol{A}$ from (13) to get

$$
p \boldsymbol{i}=\boldsymbol{V} \cdot \boldsymbol{\Lambda} \cdot \boldsymbol{V}^{-1} \boldsymbol{i}+\boldsymbol{u}
$$

By rearrangement, (17) can be written as a system of decoupled equations

$$
p \boldsymbol{Z}=\boldsymbol{\Lambda} \cdot \boldsymbol{z}+\boldsymbol{V}
$$

where $\boldsymbol{z}=\boldsymbol{V}^{-1} \boldsymbol{i}$ is the vector of the modal currents and $\boldsymbol{v}=\boldsymbol{V}^{-1} \boldsymbol{u}$ is the constant modal forcing vector.

The solution of each modal current of (18) is [13]

$$
z_{i}(t)=z_{i}(0) e^{-\lambda_{i} t}+z_{i}(\infty)\left(1-e^{-\lambda_{i} t}\right)
$$

where $z_{i}(0)$ and $z_{i}(\infty)$ are its initial and steady state values.

By inverse transformation, we get the dq currents:

$$
\boldsymbol{i}(t)=\sum_{i=1}^{n} \boldsymbol{V}_{i} c_{i} e^{-\lambda_{i} t}+\sum_{i=1}^{n} \boldsymbol{V}_{i} d_{i}\left(1-e^{-\lambda_{i} t}\right)
$$

where: $V_{i}$ is the column i of the matrix $V$

$$
\begin{aligned}
& c_{i}=z_{i}(0)=\boldsymbol{W}_{i} \cdot \boldsymbol{i}(0) \\
& d_{i}=z_{i}(\infty)=\boldsymbol{W}_{i} \cdot \boldsymbol{i}(\infty)
\end{aligned}
$$

and $W_{i}$ is the row $i$ of the inverse of matrix $V$ : 


$$
\boldsymbol{W}=\boldsymbol{V}^{-1}
$$

\section{The Numerical Tests}

\subsection{Symmetrical Three Phase Short Circuit of Unloaded Generator [14]}

In case of an unloaded generator, initially all winding currents are zero except the field.

$$
\boldsymbol{i}(0)=\left[\begin{array}{lllllll}
0 & 0 & 0 & i_{f o} & 0 & 0 & 0
\end{array}\right]^{t}
$$

where

$$
i_{f o}=v_{q o} / L_{a f}
$$

At the steady state all stator and rotor windings are shorted except the field.

$$
\boldsymbol{V}(\infty)=\left[\begin{array}{lllllll}
0 & 0 & 0 & E_{f} & 0 & 0 & 0
\end{array}\right]^{t}
$$

where

$$
E_{f}=i_{f o} R_{f}
$$

At equilibrium $p \boldsymbol{i}=\boldsymbol{O}$, so (13) will become

$$
\boldsymbol{A} \cdot \boldsymbol{i}(\infty)+\boldsymbol{u}=0
$$

from which the steady state currents can then be derived

$$
\boldsymbol{i}(\infty)=\boldsymbol{A}^{-1} \cdot\left(\boldsymbol{L}^{\mathrm{com}}\right)^{-1} \cdot \boldsymbol{V}(\infty)
$$

As an illustration, we compare ATP simulation with CS simulation. In Table 1 we present the synchronous machine parameters. Those resistances and inductances are the result of conversion of the standard synchronous machine test data [11] by ATP. In ATP library, the standard synchronous machine is the default. By examining Figure 1, we see that the ATP and CS simulations are practically similar at early cycles. In enlarged drawing in Figure 2, we see deviation in fact begins to creep at later cycles. Time step of the ATP is set to $1 \mu$ seconds. Larger time steps that produce larger deviations are also tried and for clarity reason the results are not shown. 
Table 1 Synchronous machine data

\begin{tabular}{rrrrrrrr}
\hline$L_{f}$ & 6176.50 & $L_{h}$ & 6044.76 & $L_{a k}$ & 6044.76 & $R_{a}$ & 0.1728 \\
$L_{a f}$ & 340.00 & $L_{g}$ & 6133.89 & $L_{k}$ & 6525.64 & $R_{h}$ & 10.877 \\
$L_{f h}$ & 6024.10 & $L_{a g}$ & 228.43 & $L_{o}$ & 0.7488 & $R_{g}$ & 2.6471 \\
$L_{d}$ & 10.31 & $L_{g k}$ & 5733.78 & $R_{o}$ & 0 & $R_{k}$ & 52.312 \\
$L_{a h}$ & 240.00 & $L_{q}$ & 9.8496 & $R_{f}$ & 0.3708 & & \\
\hline
\end{tabular}

Machine ratings: $100 \mathrm{MVA}, 24 \mathrm{kV}$.

Terminal voltage: $19.6 \mathrm{kV}$ ( maximum line to neutral)

Fault data: $R_{d}^{f}=R_{q}^{f}=0.576 \Omega$ and $\omega L_{d}^{f}=\omega L_{q}^{f}=1.152 \Omega$

Neglecting the mechanical dynamic, we set the frequency constant at its rating.

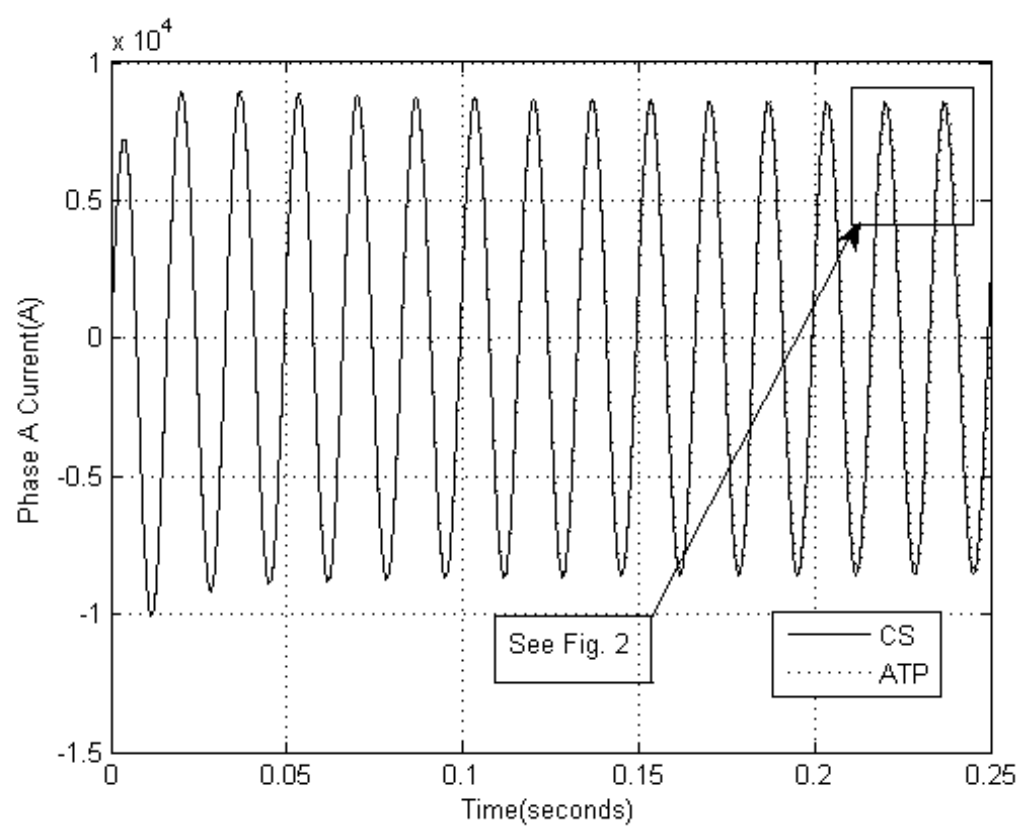

Figure 1 Short circuit on unloaded generator. 


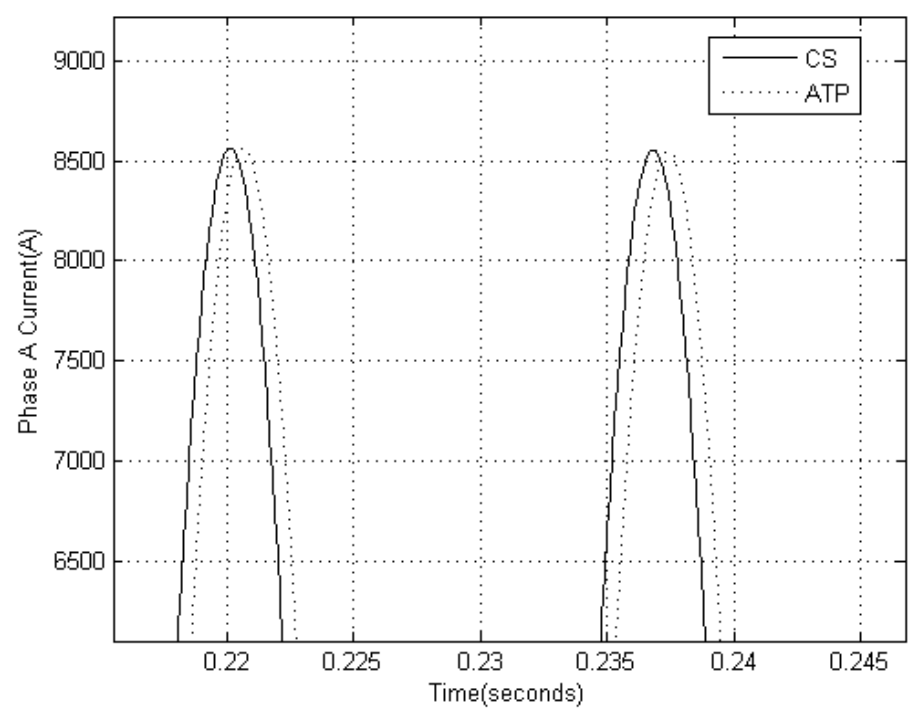

Figure 2 Zoomed Peaks of last cycle.

\subsection{Symmetrical Three Phase Short Circuit of Loaded Generator} [14]

Assuming the terminal voltages as:

$$
\left.\begin{array}{l}
v_{a}=\sqrt{2 / 3} V \sin (\omega t+\delta) \\
v_{b}=\sqrt{2 / 3} V \sin \left(\omega t+\delta-\frac{2 \pi}{3}\right) \\
v_{c}=\sqrt{2 / 3} V \sin \left(\omega t+\delta+\frac{2 \pi}{3}\right)
\end{array}\right\}
$$

Defining

$$
\theta=\omega \cdot t+\delta
$$

Therefore, $\mathrm{d}$ - and q-axis components of the terminal voltages can be calculated as

$$
v_{d o}=V \sin \delta \text { and } v_{q o}=-V \cos \delta
$$

At initial steady state, $i_{h o}=i_{g o}=i_{k o}=0$, then from (1) we have: 


$$
\left.\begin{array}{r}
\omega \psi_{q o}+i_{d o} R_{a}=v_{d o} \\
-\omega \psi_{d o}+i_{q o} R_{a}=v_{q o}
\end{array}\right\}
$$

Using relation (2), we substitute $\psi_{d o}$ and $\psi_{q o}$ from (33)

$$
\left.\begin{array}{c}
\omega L_{q} i_{q o}+R_{a} i_{d o}=v_{d o} \\
-\omega L_{d} i_{d o}+R_{a} i_{q o}=v_{q o}-\omega L_{a f} i_{f}
\end{array}\right\}
$$

From (34) we conclude

$$
\left[\begin{array}{c}
i_{d o} \\
i_{q o}
\end{array}\right]=\left[\begin{array}{cc}
-\omega L_{d} & R_{a} \\
R_{a} & \omega L_{q}
\end{array}\right]^{-1}\left[\begin{array}{c}
v_{q o}-\omega L_{a f} \frac{E_{f}}{R_{f}} \\
v_{d o}
\end{array}\right]
$$

The initial current condition is then:

$$
\boldsymbol{i}(0)=\left[\begin{array}{lllllll}
i_{d o} & i_{q o} & 0 & i_{f o} & 0 & 0 & 0
\end{array}\right]^{t}
$$

At the steady state, the condition is the same as in the unloaded generator.

In Figure 3, we see the short circuit transient in phase a as calculated by ATP and CS with the generator being loaded initially to have an angle $\delta=-20^{\circ}$. We find the same situation as in the unloaded case; the two curves are almost indistinguishable at early cycles and slowly deviating from one another only in later cycles.

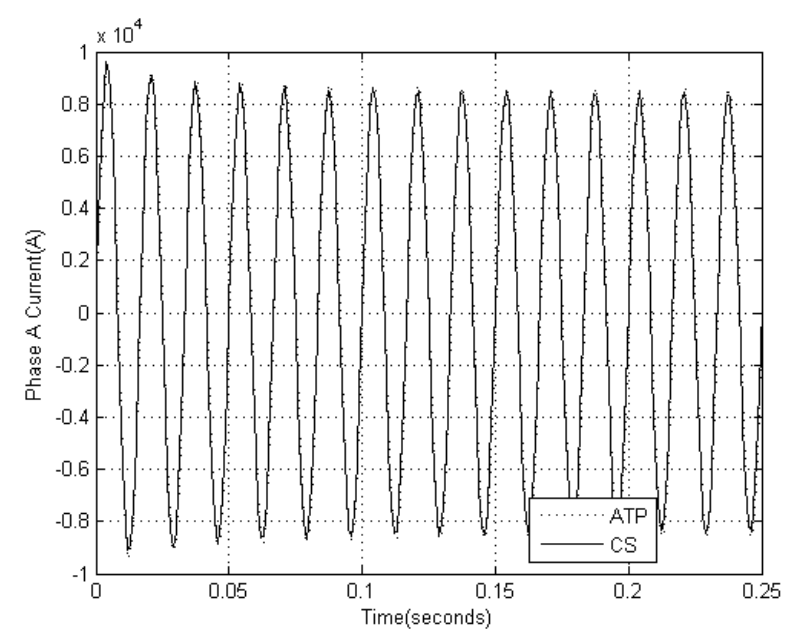

Figure 3 Short circuit on loaded generator. 


\section{Discussion}

With regard to speed, accuracy and stability, closed form solution methodology has of course overwhelming superiority over discretization based method. However, there is still restriction on its general application because of yet unsolved problems such as nonlinearity and unbalanced conditions. Of course, nonlinearity denies the application of the superposition principle at its very base. However, like nonlinearity in other problems, space for creativity is always open. An efficient way to incorporate nonlinearity into the superposition based algorithm will be much expected. We leave it as a topic of future research. Likewise, unbalanced condition is another constraint to the application of superposition principle. The fault and machine composition will no longer be trivial as in balanced fault. In dq domain the resistance and inductance matrices of the fault are no longer diagonal. Even worse it becomes time-variant. Quite likely, resolution of those problems within comparable efficiency will determine the acceptance of the proposed methodology as a general short circuit transients analysis tools in EMTP society.

Application of the method to short circuit simulation in large multi-machines power system is of course very much expected. The success of this enterprise will provide a more accurate evaluation tool than the existing static methodology as stipulated in various standards like ANSI/IEEE or IEC, or more efficient than the discretization based EMTP. Results on this will be reported in companion paper.

\section{Conclusion}

The closed form solution of the short circuit transients in synchronous machine has been derived. The simulations using CS and ATP are compared and the ATP result is closer to the CS result as the time step becomes smaller. This fact shows that the CS result is the limit a discretization based method can ultimately achieve.

\section{Acknowledgment}

The author would like to thank PT PLN Indonesia for supporting this work.

\section{References}

[1] Electro-Magnetic Transients Program (EMTP) Theory Book, EMTP 1987.

[2] Dommel, H.W., Digital computer solution of electromagnetic transients in a single and multiphase networks, IEEE Trans. Power App. Syst., PAS-88(4), pp. 388-399, Apr. 1969. 
[3] Dommel, H. W., Nonlinear and Time-varying Elements, IEEE Trans. Pwr. App. Syst., PAS-90(6), pp. 2561-2567, Nov./Dec. 1971.

[4] Cui, Yu., Dommel, Herman W. \& Xu, Wilsun., A Comparative Study of Two Synchronous Machine Modeling Techniques for EMTP Simulation, IEEE Trans. On Energy Conversion, 19(2), pp. 462-463, June 2004.

[5] Brandwajn, V., Representation of Magnetic Saturation in the Synchronous Machine Model in an Electro-Magnetic Transients Program, IEEE Trans. Power App. Sys., PAS-99(5) Sept/Oct 1980.

[6] Chan, K.H., Acha, E., Madrigal, M. \& Parle, J.A., The use of direct timephase domain synchronous generator model in standard EMTP-type industrial packages, IEEE Power Eng. Rev., pp. 63-85, June 2001.

[7] Marti, J.R. \& Louie, K.W. A phase-domain synchronous generator model including saturation effects, IEEE Trans. Power Sys., 12, pp. 222229, Feb. 1997.

[8] Cao, X., Kurita, A., Mitsuma, H., Tada, Y. \& Okamoto, H., Improvements of Numerical Stability of Electromagnetic Transient Simulation by Use of Phase-Domain Synchronous Machine Models, Elec. Eng, in Japan, 128(3), pp. 53-62, 1999.

[9] Wang, Liwei \& Jatskevich, Juri, A Voltage-Behind-Reactance Synchronous Machine Model for the EMTP-Type Solution, IEEE Trans. Pow. Syst., 21(4), pp. 1539-1549, Nov. 2006.

[10] Wang, Liwei., Jatskevich, Juri \& Dommel, Herman W., Re-examination of Synchronous Machine Modeling Techniques for Electromagnetic Transient Simulations, IEEE Trans. Pow. Syst.,22(3), pp. 1221-1230, Aug. 2007.

[11] Sianipar, Gibson H.M. Superposition-Based Fault Analysis-The Exact Maximum Asymmetric Current, ASEAN Symposium on Power and Energy Systems (ASPES), Hua Hin, Thailand, Sept. 2009.

[12] Kundur, P., Power System Stability and Control. New York: McGrawHill, 1994.

[13] Chua, Leon O., Desoer, Charles A. \& Kuh, Ernst S., Linear and Nonlinear Circuits, McGraw-Hill, 1987.

[14] Padiyar, K.R., Power System Dynamics. Stability and Control. Singapore, John Wiley \& Sons, 1996.

[15] IEEE Subsynchronous Resonance Task Force of the Dynamic System Performance Working Group, Power System Engineering Committee, First benchmark model for computer simulation of subsynchronous resonance, IEEE Trans. Power App. Sys., PAS-96, pp. 1565-1570, Sept/Oct 1977.

[16] Marti, J.R. \& Linares, L.R., Real-Time EMTP-Based Transients Simulation, IEEE Trans. Pow. Sys., 9(3), pp. 1309-1317, Aug. 1994. 